\title{
A Decision Process Model to Support Migration to Cloud Computing
}

\begin{abstract}
Migration to cloud computing is a strategic organisational decision that can affect performance, productivity, growth, as well as increase competitiveness. The decision to migrate is usually complicated and dynamic due to the immaturity and the still evolving nature of the cloud environment. Although there have been many proposed methods for supporting the migration, no systematic decision process exists that clearly identifies the main steps and explicitly describes the tasks to be performed within each step. In this paper, a decision-making process model, based on a two-stage survey, is proposed. The model guides decision makers through a step-by-step approach, aiding them with their decisions for cloud migration. It offers a preliminary structure for developing a cloud knowledge-based decision support system. The model was evaluated by a group of cloud practitioners. The analysis demonstrates a high level of acceptance with regard to the structure, tasks involved and issues addressed by it.
\end{abstract}

Keywords. Cloud computing, cloud migration, decision-making process, cloud decision support, cloud knowledge-based system

\section{Introduction}

Cloud computing has emerged as a paradigm to deliver on-demand IT resource which has opened up new opportunities for enterprises to enhance the design of their resources and architectures, and to rationalise their information systems expenditure (Garg, 2013). Cloud computing offers new capabilities and business models that would improve profits, and also produce further competitive differentiation. These have changed the way enterprises organise, balance, maintain, and pay for their systems (Dhinesh Babu et al., 2014).

Omerovic et al. (2013) pointed out that one of the major challenges regarding cloud services is that they usually vary in their business models, functionality, quality of service, cost, etc. The range of cloud-based services offered is also growing with the emergence of new cloud service providers. In addition, the heterogeneity of IT systems and business processes within organisations, and the changes to their requirements have increased the complexity of decision-making in respect of migration to cloud-based solutions.

Companies often decide to migrate their IT resources to the cloud due to many organisational and technical benefits. However, as cloud computing represents a fairly new paradigm, there is still a significant number of issues and risks which still remain to be resolved so that cloud services can be implemented properly with ease (Sharma and Abrol, 2013). It involves amongst other things, concerns about loss of privacy, disruption to business processes, interoperability, data integrity, compliance violation, application portability, contracting issues, business continuity, staff productivity, and security issues.

Although, there have been many proposed methods for supporting migration to the cloud, no systematic decision-making process exists that clearly identifies the main steps and explicitly describes the tasks to be performed within each step. Therefore, this paper aims to fill this need by proposing a model to support the decision-making process to migrate legacy systems to the cloud. The methodology employed for developing the model comprises three main phases: a two-stage survey, application of a generic decision-making process to structure the activities, and evaluating the model by analysing views of a group of cloud practitioners. The survey identified issues faced by decision makers and activities that need to be performed. It reveals that in order for organisations to make informed decisions to migrate 
to the cloud, it is essential that there is an appropriate migration plan, as well as a clear understanding of the new environment. Further, a service suitability analysis as well as providers' evaluation should also be carried out prior to the selection and implementation processes.

The model provides a structure with guidelines that cover the whole decision making process for migration. The proposed model can lead to the development of a cloud Knowledge-Based Decision Support System (KBDSS). KBDSSs use dedicated knowledgebases for problem-solving which refer to a particular domain and an understanding of the problems within this domain, and certain expertise in solving problems of this kind (Ada et al., 2015).

This paper is structured as follows. Section 2 summarizes the current situation with regard to the cloud migration process in two sub-sections: firstly it looks at the existing decision support systems, and secondly it identifies the need for a cloud migration decision process. Based on the analysis of the two-stage survey, Section 3 discusses the challenges of migrating to the cloud. Section 4 examines the proposed decision process model in detail. Section 5 provides an evaluation of the proposed process. Finally, the conclusions are drawn, and future work discussed, in Section 6.

\section{Background}

\subsection{Cloud migration decision support systems}

Many Decisions Support Systems (DSSs) to assist organisations with migrating to cloud computing have been proposed. They mostly concentrated on the evaluation and selection of cloud providers with cost being the main factor.

The suggestions made in (Khajeh-Hosseini et al., 2012), (Menzel et al., 2014), (GarcíaGalán et al., 2015), and (Santiago, 2015) particularly focus on the selection of the providers for Infrastructure as a Service (IaaS). Khajeh-Hosseini et al. (2012) developed a cloud adoption toolkit that aids decisions on the suitability of the technology, consumption of energy, cost, impacts of stakeholders and operational viability. It incorporates two decision support tools: cost calculation and risk-benefit analysis. Menzel et al. (2014) developed the CloudGenius framework that provides a multi-criteria approach in decision support for selecting providers for IaaS to migrate a web server to the cloud. It allows users to define multiple quantitative and qualitative requirements that are then matched against a knowledgebase of cloud service providers. García-Galán et al. (2015) focused on supporting the decision-making for selecting the most suitable cloud configuration. Whilst Santiago (2015) focused on the evaluating of IaaS providers based on their performance and costs for migrating a workflow-based simulation environment.

Andrikopoulos et al. (2013a) and Rai et al. (2014) focused on supporting migration of applications to the cloud. The characteristics of applications (Andrikopoulos et al., 2013a) and the need for adaptation (Andrikopoulos et al., 2013b) to operate in the cloud environment were also taken into account. Andrikopoulos et al. (2013a) proposed a decision support system to select the cloud offerings for migrating applications that best matched the parameters defined by users. Rai et al. (2014) considered a number of key business and technical factors that need to be analysed that would help to select appropriate applications for migrating.

In an effort to standardise measuring and comparing cloud services, Garg (2013) designed the Service Measurement Index (SMI). It uses an analytical hierarchy process mechanism based on the quality of service attributes defined by the ISO, to rank cloud providers. The 
SMI has been considered to be a standard for service assessment and comparison within the Cloud Standards Coordination (CSC, 2013).

Limited studies have considered the support required for the analysis of the requirements and characteristics of existing company-based IT resources and also the feasibility of migrating to the cloud environment. Misra and Mondal (2011) proposed four key characteristics of company based-IT resources which should be considered during the analysis for migration. They are: size of the IT resources, the utilisation pattern of the resources, sensitivity of the data they are handling, and criticality of the service.

Despite of the developments of these DSSs, many challenges still remain, both from the research and technical perspectives (Gonidis, 2014). The majority of the existing DSSs do not support the assessment of business processes and relevant applications, nor do they provide information for the analysis of the impact of the chosen cloud services. Although, evaluation of providers and their appropriate selection are critical, making an informed decision to migrate requires analysing a wide range of factors at earlier stages of a decision process. Companies should become fully aware of the cloud capabilities, guiding principle, the services offered and their potentials before coming to a decision.

Supporting these require a process for collecting information to assist each phase of the decision making. Some of the cloud DSSs utilise knowledge-bases that provide cloud offerings along with their pricing policies as discussed in (Menzel et al., 2014) and (Andrikopoulos et al., 2013a). However, to make migration decision, a wider range of information such as cloud capabilities, services offered, pricing schemes, etc., should be considered. This will aid organisations to identify opportunities and services that can help to improve business processes and operation.

The range of information required to be considered for migration is increasing due to the development of the technology and expansion of the services offered. Also the number of proposed tools which are becoming available is increasing at a bewildering rate. What support is available to guide organisations through the process systematically? The next section reviews two attempts for providing a systematic decision process for migration to cloud computing as well as a generic decision-making process model.

\subsection{A systematic decision process}

Beserra et al. (2012) proposed a step-by-step cloud decision process to support migration of legacy applications to the cloud which is organised into nine activities. The process relies on the creation of template based profiles characterising the organisation, the target legacy application and candidate cloud providers, which are then cross-analysed to help in identifying and possibly resolving critical constraints that may hinder the migration.

However, this process has a number of limitations. It does not include an evaluation of the cloud environment which is essential to ensure an adequate understanding of the new environment for organisations prior to migration. The process is also primarily focused on supporting software developers whereas cloud migration is a business driven decision that involves organisational risks much more than just the technical aspects. Further, the analysis of applications do not include major tasks, such as integration requirements, especially in the case of hybrid deployment scenarios, the main standards and regulation which might be required for some applications.

In an attempt to enhance the cloud migration process, Jamshidi et al. (2013) introduced the Cloud-RMM reference model. The model is based on the analysis of 23 existing cloud migration frameworks and decision support systems. It conceptualised a process-centric view for state-of-the-research that was classified into four processes and 20 migration tasks. 
Jamshidi et al. concluded there was a need for a comprehensive migration framework that supports the migration process.

To ensure a systematic process for decision making, Simon (1977) designed a generic model to address decisions that are highly unstructured, complex, involve risks and that would have an impact on the future of an organisation. It includes three major phases: Intelligence, Design, and Choice. The process starts with the Intelligence phase where the "reality" of the organisation is examined. It involves problem identification and information gathering activities about the societal, the competitive, and the organisational environments. In the Design phase, a model is constructed that simplifies the organisation's "reality" and identifies relationships between variables, as well as setting the criteria for evaluating alternative courses of action. The Choice phase is to select the most appropriate alternative course of action based on the criteria identified in the Design phase. Successful implementation results in the delivery of the required values to meet the organisation's strategic vision. Failure requires a return to an earlier phase of the process. Figure 1 shows this decision-making process model.

Figure 1 Simon's decision making process

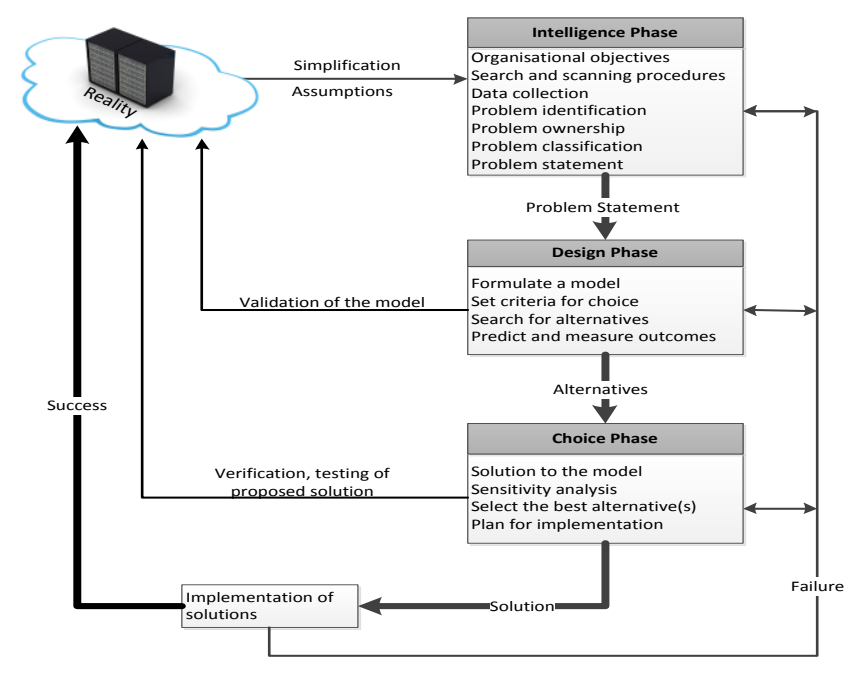

The availability of information is particularly fundamental in the first two phases of a decision-making because alternatives can only be chosen where there are sufficient information about them and that they can be documented. Unavailability of information is the weakness in many of the existing DSSs, as many designers place emphasis on the models that they build into their systems rather than the significance of the information fed into them (Diniz et al., 2008).

In this paper, we address the need for a comprehensive support for migration by proposing a cloud decision process model. Our proposed model is based on the generic decision-making process model developed by Simon. The Simon's model was selected since it covers all phases of decision making. Further, this work puts a specific emphasis on planning for migration due to the following reasons:

- Cloud migration is a strategic organisational decision that should be guided by the main organisational requirements and objectives for migration by which all further steps should be analysed accordingly,

- The immaturity and evolving nature of cloud computing which requires a proper migration strategy and analysis, 
- The large number of information sources, their levels of accessibility, reliability and cost related information present a complex data gathering and analysis problem,

- Availability of a wide range of decision support systems and cost calculation tools (some of those discussed in 2.2.1) which can create uncertainty for decision makers if they are used outside of a systematic process.

\section{Migration challenges}

A first step toward developing the decision-making process was to gain an insight into the perception of cloud users as well as cloud providers on migration challenges. A two-stage survey was employed which aimed to explore issues and challenges that users had experienced during the migration process. Concerns, requirements, and issues surrounding the process of migration to the cloud were identified.

Stage1: twelve practitioners including IT managers, security professionals, and cloud professionals (from cloud providers' perspective) were interviewed. The findings of these interviews were discussed in Alkhalil et al., (2013) and Alkhalil et al., (2014). One of the main issues raised was related to customers' lack of knowledge of the cloud environment. This has caused many issues such as unawareness of interpretability problems and costs associated with returning to the original legacy system, an overestimation of cloud-based services, as well as security and privacy concerns.

The issues and challenges raised in Stage 1 were incorporated into the questions used in Stage 2 that was based on a quantitative online survey questionnaire. The target population consisted of a 102 professionals who had been involved in cloud migration projects. Participants were from organisations of different sizes and from a divers industry sectors. They included IT managers, software engineers, system analysts, and executive managers; in addition to cloud systems researchers. The following sections provide a summary of the analysis of the responses which had outstanding results.

\subsection{Issues deterring organisations from migrating}

The participants were asked: which of the following in your view are deterring organisations from migrating to the cloud? A total of 14 reasons, as shown in table 1 were provided which might negatively affect the decisions for migrating to the cloud.

Table 1 Issues deterring from migrating

\begin{tabular}{|c|c|c|c|c|c|c|c|c|c|c|c|c|c|c|}
\hline $\begin{array}{l}\text { Answer } \\
\text { Options }\end{array}$ & 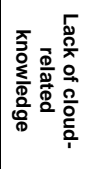 & 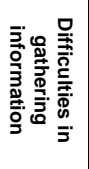 & 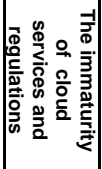 & 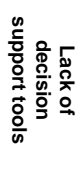 & 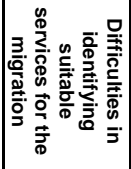 & 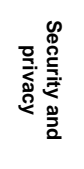 & 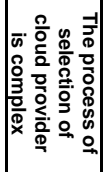 & 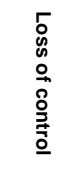 & 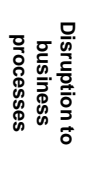 & 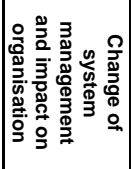 & 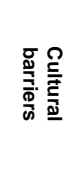 & 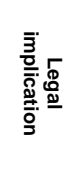 & $\underset{\underline{\underline{Z}}}{\vec{z}}$ & 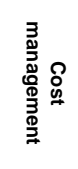 \\
\hline $\begin{array}{l}\text { Response } \\
\text { Per cent }\end{array}$ & $71.7 \%$ & $30.3 \%$ & $42.4 \%$ & $17.2 \%$ & $22.2 \%$ & $66.7 \%$ & $18.2 \%$ & $45.5 \%$ & $28.3 \%$ & $24.2 \%$ & $11.1 \%$ & $43.4 \%$ & $43.4 \%$ & $18.2 \%$ \\
\hline $\begin{array}{l}\text { Response } \\
\text { Count }\end{array}$ & 71 & 30 & 42 & 17 & 22 & 66 & 18 & 45 & 28 & 24 & 11 & 43 & 43 & 18 \\
\hline
\end{tabular}

It can be seen in Table 1 that the majority of the participants (approximately $72 \%$ ) indicated lack of cloud related knowledge as the primary factor deterring migration to cloud computing. This confirms the conclusion of stage 1 that "customers' lack of knowledge of cloud environment is a key factor that deters organisations from migrating to cloud 
computing". Security and privacy was found to be a major barrier for migration too with approximately $67 \%$ of participants selecting this reason. The loss of control (45\%) was also found to be a concern that deters some organisations from migrating to the cloud. Lack of trust in cloud computing (43.4\%) and continuing problems with the cloud-based services $(42.4 \%)$ were two other issues which had scored high in comparison to the other issues provided.

\subsection{Legal Implication}

Violation of legal compliance has become a concern for many organisations (Gleeson and Walden, 2014). In a question (see table 2) we sought the participants' views in this regard. The question was: which of the following are the main legal implications which can affect decisions to migrate to cloud computing?

Table 2 Legal Implication

\begin{tabular}{|c|c|c|c|c|c|c|c|c|}
\hline $\begin{array}{l}\text { Answer } \\
\text { Options }\end{array}$ & 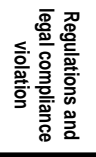 & 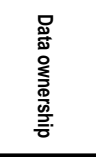 & 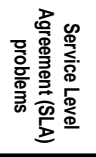 & 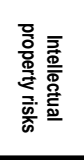 & 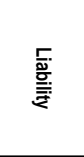 & 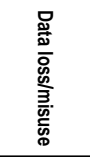 & 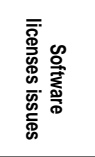 & zo \\
\hline $\begin{array}{c}\text { Response } \\
\text { Percent }\end{array}$ & $73.3 \%$ & $43.6 \%$ & $49.5 \%$ & $41.6 \%$ & $26.7 \%$ & $54.5 \%$ & $20.8 \%$ & $0.0 \%$ \\
\hline $\begin{array}{l}\text { Response } \\
\text { Count }\end{array}$ & 74 & 44 & 50 & 42 & 27 & 55 & 21 & 0 \\
\hline
\end{tabular}

As it is shown in table 2, a significant number of the participants $(73.3 \%)$ indicated that regulations and legal compliance violation are the main concerns that might affect their decisions to migrate to the cloud.

Participants also expressed concerns in respect of legal implications of their data. $54.5 \%$ of the participants indicated that concerns in case their data being lost or misused by others. About half of the participants pointed out that legal implications of SLAs had affected their decisions to migrate or not to the cloud. Further, $43.6 \%$ of the participants pointed out their concerns in respect of data ownership. About $41.6 \%$ indicated intellectual property rights as a legal concern.

\subsection{Cloud knowledge and skills}

Participants were given a list of 11 options in respect of knowledge and skills and they were asked to identify those which would be mostly required when dealing with cloud services. Table 3 show the options.

Table $3 \quad$ Cloud knowledge and skills

\begin{tabular}{|c|c|c|c|c|c|c|c|c|c|c|c|c|}
\hline $\begin{array}{l}\text { Answer } \\
\text { Options }\end{array}$ & 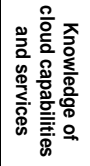 & 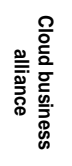 & 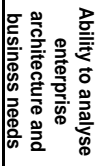 & $\begin{array}{l}\frac{0}{\circ} \\
\frac{0}{0} \\
\overline{0} \\
0.0 \\
\bar{\Xi}\end{array}$ & 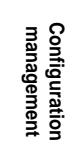 & 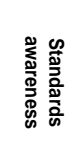 & 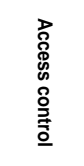 & 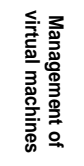 & 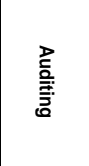 & 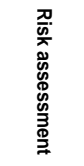 & 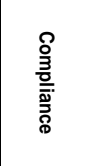 & zo \\
\hline $\begin{array}{c}\text { Response } \\
\text { Percent }\end{array}$ & $83.0 \%$ & $8.5 \%$ & $34.0 \%$ & $16.0 \%$ & $41.5 \%$ & $39.4 \%$ & $\mathbf{4 0 . 4 \%}$ & $22.3 \%$ & $29.8 \%$ & $60.6 \%$ & $34.0 \%$ & $2.1 \%$ \\
\hline $\begin{array}{c}\text { Response } \\
\text { Count }\end{array}$ & 78 & 8 & 32 & 15 & 39 & 37 & 38 & 21 & 28 & 57 & 32 & 2 \\
\hline
\end{tabular}

The vast majority of participants $(83 \%)$ believed that there is a need to expand their knowledge and skills with regards to the cloud capabilities and services. The reason could be 
due to the rapid changes of cloud providers, services and their delivery models. The wide concerns of security and privacy were indicated again by $60.6 \%$ of the participants expressing that there is a need for cloud risk assessment skills. Other skills such as configuration management (41.5\%), access control (40.4\%), standards awareness $(39.4 \%)$, and the ability to analyse enterprise architectures and business needs (34\%) are also worth of consideration.

\subsection{Quality of existing cloud-related information}

Participants' views with regards to the quality of the current cloud-related information were explored. The responses are presented in table 4.

Table 4 Quality of existing cloud-related information

\begin{tabular}{|c|c|c|c|c|c|c|c|}
\hline Answer Options & Very low 1 & 2 & 3 & 4 & Very high 5 & $\begin{array}{c}\text { Rating } \\
\text { Average }\end{array}$ & $\begin{array}{l}\text { Standard } \\
\text { Deviation }\end{array}$ \\
\hline Availability & $\begin{array}{c}1.12 \% \\
1\end{array}$ & $\begin{array}{c}2.25 \% \\
2\end{array}$ & $\begin{array}{c}25.84 \% \\
23\end{array}$ & $\begin{array}{c}41.57 \% \\
37\end{array}$ & $\begin{array}{c}29.21 \% \\
26\end{array}$ & 3.96 & 0.86 \\
\hline Sufficiency & $\begin{array}{c}4.44 \% \\
4 \\
\end{array}$ & $\begin{array}{c}6.67 \% \\
6 \\
\end{array}$ & $\begin{array}{c}38.89 \% \\
35 \\
\end{array}$ & $\begin{array}{c}34.44 \% \\
31 \\
\end{array}$ & $\begin{array}{c}\mathbf{1 5 . 5 6 \%} \\
14 \\
\end{array}$ & 3.50 & 0.98 \\
\hline Accessibility & $\begin{array}{c}1.14 \% \\
1\end{array}$ & $\begin{array}{c}3.41 \% \\
3\end{array}$ & $\begin{array}{c}32.95 \% \\
29\end{array}$ & $\begin{array}{c}38.64 \% \\
34\end{array}$ & $\begin{array}{c}23.86 \% \\
21\end{array}$ & 3.81 & 088 \\
\hline Accuracy & $\begin{array}{c}5.56 \% \\
5\end{array}$ & $\begin{array}{c}26.67 \% \\
24\end{array}$ & $\begin{array}{c}36.67 \% \\
33\end{array}$ & $\begin{array}{c}22.22 \% \\
20\end{array}$ & $\begin{array}{c}8.89 \% \\
8\end{array}$ & 3.02 & 1.03 \\
\hline Consistency & $\begin{array}{c}\mathbf{1 4 . 4 4} \% \\
13\end{array}$ & $\begin{array}{c}27.78 \% \\
25\end{array}$ & $\begin{array}{c}35.56 \% \\
32\end{array}$ & $\begin{array}{c}18.89 \% \\
17\end{array}$ & $\begin{array}{c}3.33 \% \\
3\end{array}$ & 2.69 & 1.04 \\
\hline Understandability & $\begin{array}{c}4.49 \% \\
4\end{array}$ & $\begin{array}{c}21.35 \% \\
19\end{array}$ & $\begin{array}{c}48.31 \% \\
43\end{array}$ & $\begin{array}{c}\mathbf{2 1 . 3 5 \%} \\
19\end{array}$ & $\begin{array}{c}4.49 \% \\
4\end{array}$ & 3.00 & 0.89 \\
\hline Timely & $\begin{array}{c}3.33 \% \\
3\end{array}$ & $\begin{array}{c}\mathbf{1 8 . 8 9} \% \\
17\end{array}$ & $\begin{array}{c}48.89 \% \\
44\end{array}$ & $\begin{array}{c}22.22 \% \\
20\end{array}$ & $\begin{array}{c}6.67 \% \\
6\end{array}$ & 3.10 & 0.90 \\
\hline Diversity & $\begin{array}{c}3.37 \% \\
3\end{array}$ & $\begin{array}{c}7.87 \% \\
7\end{array}$ & $\begin{array}{c}24.72 \% \\
22\end{array}$ & $\begin{array}{c}47.19 \% \\
42\end{array}$ & $\begin{array}{c}16.85 \% \\
15\end{array}$ & 3.66 & 0.96 \\
\hline Complexity & $\begin{array}{c}3.37 \% \\
3\end{array}$ & $\begin{array}{c}3.37 \% \\
3\end{array}$ & $\begin{array}{c}28.09 \% \\
25\end{array}$ & $\begin{array}{c}42.70 \% \\
38\end{array}$ & $\begin{array}{c}\mathbf{2 2 . 4 7} \% \\
20\end{array}$ & 3.78 & 0.95 \\
\hline
\end{tabular}

The analysis shows that there is a high level of availability of cloud related information. More than two-thirds (approximately 70\%) of the participants rated the availability of cloudrelated information as high or very high while only less than $3 \%$ indicated that there is limited information. Accessibilities and sufficiency of cloud related information were also scored highly rated by average weights of 3.81 and 3.50 respectively. Moreover, participants indicated that cloud-related information was reasonably accurate (3.02) and could be understood (3.0).

Despite the high rating of the aforementioned, participants did indicate that there is a problem with consistency in the cloud related information (with an average rate of just 2.29), a high level diversity of cloud-related information (3.66) and also it is complex (3.78).

Although many organisations believe that cloud-related information can easily be obtained and to some extent it is reliable, they do not find it easy to develop an understanding about the cloud environment. This can be due to the diversity of information sources and their complexity. This could be the reason for the issue of limited knowledge of customers in respect of the cloud environment.

\subsection{The need for cloud knowledge-base}

In this study we propose the use of a knowledge-base that will provide a repository for all relevant information and decision assisting tools. The participants were asked "To which extent do you think there is a need for a cloud knowledge-base to overcome the lack of customers' cloud-related knowledge?" The vast majority of respondents (85\%) indicated the need for a cloud knowledge-base as important while only (12.5\%) expressed it is not important or they were not sure. 


\section{A cloud migration decision process model}

Following on Simon's decision process model (Simon, 1977) and the outcome of the twostage survey discussed in section 3, a decision process model for migrating to the cloud is proposed. The process is focused on the identification and analysis of relevant areas that might influence the decision to migrate. Particularly, the model centred on the tasks required during the intelligence and design phases which have been neglected by existing DSSs.

The model consists of four main components: Information Sources, a Knowledge-Base Decision Support System (KBDSS) platform, and a Cloud Migration Process, as shown in Figure 2.

Figure 2 The Cloud migration decision process model: an overview

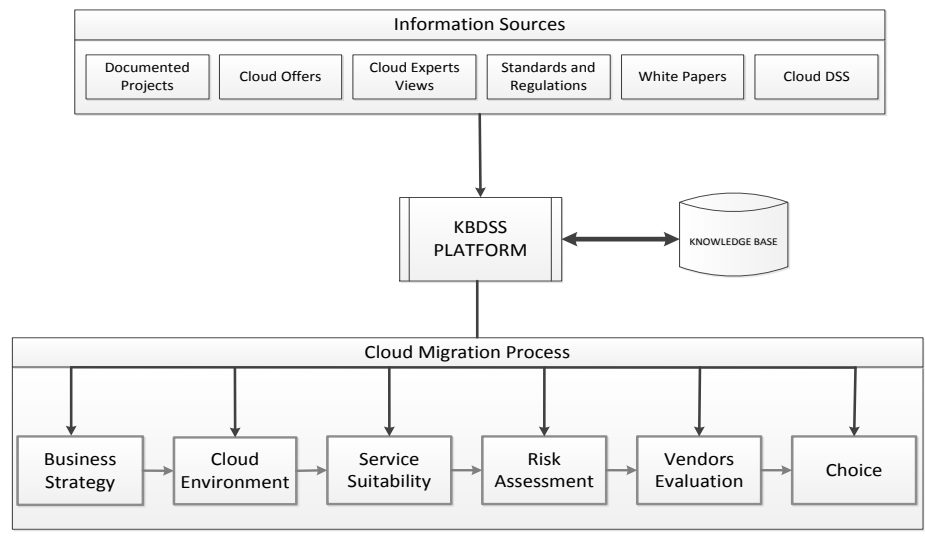

The sources for gathering information may include: documented projects, cloud services offered, cloud experts' views, relevant standards and regulations, vendors' white papers, and existing cloud DSSs. A KBDSS platform can be utilised to structure the gathered information before storing them in the knowledge-base. It will also be used as an access management point to and from the knowledge-base. The knowledge-base is a major integrated part in this model that stores the gathered information to assist each phase of the migration process. It may include facts about cloud environment and procedural information (cloud rules). The knowledge-base could be constructed through the use of existing intelligence techniques for knowledge acquisition and an integration of cloud DSSs and tools.

The cloud migration process block comprises six main steps. They are: business strategy, cloud environment, service suitability, risk assessment, vendors' evaluation, and implementation. These steps would support the decision-making process by guiding decision makers through a structured hierarchy of tasks. Subsequently, using the multiple analytical techniques to analyse and select alternative solutions.

Figure 3 shows the sequence and the inter-relationship between these steps, as well as the tasks to be carried out in each step. The process starts by examining the internal environment and identifying the organisational objectives for migration. The next step is to recognise the cloud guiding principles and capabilities. This step should be carried out with reference to the knowledge-base that should provide structured information about cloud offers, pricing policies, security polices, performance monitoring, compliance to regulators, and support. Subsequently, the process moves to the design phase that involves two main steps: an identification of the suitable services to be migrated and a risk assessment for each candidate service. The tasks performed during this stage ensure that the requirements and characteristics of business services are suitable for the cloud environment. Then, decision makers are required to evaluate the cloud venders to select the appropriate providers. In this model, the 
SMICloud for cloud providers' ranking and evaluation developed by Garg (2013) has been adopted. The final step in this process is to make the choice for the most appropriate cloud provider and then implementation.

In contrast to the processes proposed by Beserra et al. (2012) and Jamshidi et al. (2013), to ensure appropriate decisions are made, the range of information required for consideration in this model is augmented by the existing cloud migration tools for applications analysis and selection of providers. This information can be provided to decision makers through a knowledge-base. Decision makers will be guided through the key decisions which they need to consider by going through a number of tasks which are identified in the intelligence phase.

Unlike the previously discussed DSSs, this model was not designed to support the migration of a specific service. Therefore, the model can be applied in general contexts and various domains. Additionally, the model includes a wider analysis than just evaluation of the technical aspects, for example legal issues and the impact of organisational culture were considered. Furthermore, the systematic structure in this model will allow decision makers to effectively exploit a wide range of cloud migration tools and decision support systems that may be required in the later phases (Design and Choice). The following sub-sections provide detailed discussions on decisions involved in the process and the tasks to be performed.

\section{Figure 3 Cloud migration decision process model}

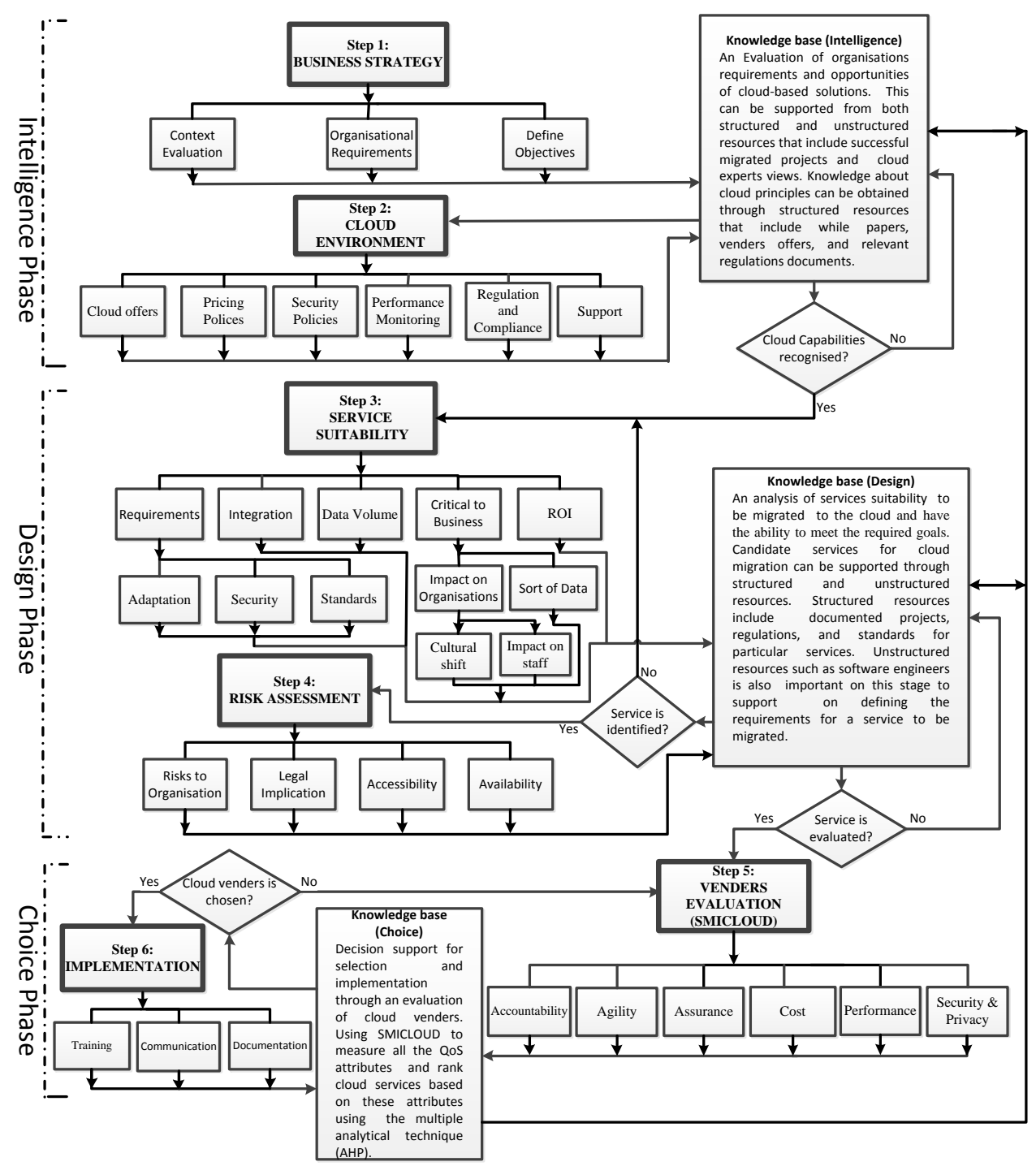




\subsection{Business strategy}

Defining the business strategy and developing an understanding of the cloud environment constitute the intelligence phase of the decision-making process which can be supported through the intelligence level of the knowledge-base. This phase includes activities for gathering information from the environment in which the system is currently operating in to identify requirements, market trends, and then to define the objectives. It also includes information gathering from the new environment, to identify its main guidelines and opportunities. The process starts by formulating the business strategy for migration. Internal and external environments should be considered to formulate a strategy to gain a competitive edge. Large organisations may follow a strategy that involves starting their own private cloud with a potential to move to a public cloud, if required in the future.

\subsubsection{Context evaluation}

Defining the business strategy for migration begins with an evaluation of the current organisational environment and the analysis of requirements and objectives. It includes understanding of organisation's characteristics, assessment of the organisational innovation culture, trends and the competitive environment. Easy access to data, information, performance metrics, customer behaviour, and market trends is vitally important in today's global competitive nature. These tasks can be supported by business intelligence techniques such as data mining, data warehousing, score-carding, dash-boarding, and financial analysis.

\subsubsection{Organisational requirement}

After gathering information about the organisational context, the next task is to identify the organisational requirements. The purpose of this task is to detect potential organisational constraints that might affect cloud migration decisions; such constraints should be the central for any further analysis. Organisational requirements may be legal, financial, policy, level of service, guideline or administrative in nature.

\subsubsection{Define the organisational objectives}

Organisations should define the exact objectives for migration. In addition to cost reduction, migration to cloud computing can be a strategic decision for organisations to enhance business innovations through enhancing scalability, flexibility, and time to market. The need for testing and staff training before deploying a new system can be an issue for some organisations. In the cloud, new business processes can be designed and tested through the cloud environment before their official deployment. Organisations can have a replica environment running in parallel, in the cloud and keeping the in-house legacy system running as normal. If the new system delivers the sought strategic values, organisations can then backup their legacy data and have it available, starting the new system with a fresh configuration and then moving traffic across. This approach of deployment can significantly reduce the risk of impact as the two versions would be running at the same time with the ability of moving data across the two environments. For organisations that require remote access, the in-house system can initially be used as a back-up and the cloud version as the live system due to the high bandwidth capacity offered by the cloud. 
The second key step is to identify the guiding principle of the cloud and to recognise the cloud's capabilities. The purpose of this step is to aid decision makers in acquiring the knowledge they need prior to the migration. Six main areas are identified which are depicted in Figure 3 as tasks that would aid cloud customers to become more familiar with the cloud environment. They are: cloud offerings, pricing policies, security policies, performance monitoring, compliance with regulations, and support. Organisations may complete this step by using the knowledge-base, which can provide the necessary information with regard to the cloud environment. It also aims to enable them to identify services offered by the providers which are appropriate to their needs.

\subsubsection{Cloud offers}

The levels of details of functionality provided to cloud users for managing and controlling the underlying cloud infrastructure are varied in the three cloud service models (SaaS, PaaS, and IaaS) (Sahandi, 2013). Organisations need to develop knowledge of how their services can be managed in the different cloud models. Further, cloud services are usually offered by providers in a fixed pricing model (tariffs). Some organisations may have their own usage models. Therefore, they may have to adapt their services usage according to the cloud tariffs. This could be a challenge for some organisations, particularly the larger ones which usually have fixed usage models.

\subsubsection{Pricing policies}

Reducing expenditure through the deployment of cloud services is largely dependent upon the pricing models that may be compatible with the customer's behaviour (Al-Roomi et al., 2013). Cloud-based services are usually provided in different pricing schemes. Pay-as-you-go is the most commonly used in cloud computing. The price is usually calculated by applying one of the following: fixed price regardless of volume, fixed price plus per unit rate, assured purchase volume plus per unit price rate, per-unit rate with a ceiling, and per unit price (Iveroth et al., 2013). Therefore, organisations need to develop knowledge about these pricing schemes to avoid unnecessary costs.

\subsubsection{Security policies}

The security issues and responsibilities are varied in the three deployment models (SaaS, PaaS, and IaaS). This requires organisations to have full awareness of the security responsibility for each model. Confidentiality is a critical aspect for all organisations. To ensure confidentiality of migrated data in the cloud environment, organisations are required to have full details of who has accessed or requested access to their data. This signifies the importance of developing knowledge about how to track accessibility to the cloud services and the different tracking tools offered by cloud providers. Organisations need to develop knowledge security landscapes such as data protection and encryption, digital signing, identity management, authentication methods, and auditing. Information about the location of data-centres, accessibility to physical resources, administration, operation, and disaster recovery plans are also vital for organisations. Reliable access to resources is a key requirement for organisations which require a wide range of information, assurances against a number of potential threats are vital including: network vulnerability, multi-site redundancy, IP failover and secure transfer of data. 


\subsubsection{Performance monitoring}

Resource sharing techniques and virtualisation in the cloud often affect performance and degradation causing computing nodes to respond slowly or even become temporarily unavailable (Meng et al., 2012). Moreover, cloud services are usually hosted over distributed web servers, application servers and data-bases to achieve high scalability and reliability (Garg, 2013). These issues have an impact on monitoring which is a necessary building block for many distributed applications and services hosted in cloud data-centres. It includes safeguarding performance, consumptions and cost, end-user experiences, detection of attacks, data-centre-wide profiling, availability, measurement of service outages, and instant message delivery. Therefore, it is vital for organisations to develop knowledge on how to monitor performance when using cloud services, the different levels of monitoring and also on the tools that are available for systems monitoring.

\subsubsection{Compliance to regulations}

It is critical for organisations to ensure the validity of their cloud services. Achieving this requires compliance information gathering from different regulators. These include national and international standards for a particular service and the cloud providers' regulations. The gathered compliance information will then help organisations to design and implement control objectives to meet the relevant compliance requirements.

\subsubsection{Support}

Support services are provided at different levels and costs, thus organisations need to identify the level of support they require and whether this support is available at an acceptable cost.

\subsection{Services suitability}

Once the objective is finalised and the cloud standards and capabilities are recognised, the migration decision moves to the design phase. The purpose of this phase is to aid the selection of the services for migration. This phase involves two main steps: (a) an identification of the services that are suitable to be migrated; (b) performing a risk assessment for each candidate service.

The process of evaluating the suitability of a service includes: selection the service's requirements, integration, data volume, criticality to business, and a ROI calculation. These tasks are performed during this stage to ensure that the requirements and characteristics of business services are suitable to be supported by the cloud environment. The design level of the knowledge-base can provide information to support in performing these tasks. When a service is selected it is critical to conduct a risk assessment for each of the selected service, including the risks to the organisation, the legal implication, the security threats and their consequences.

\subsubsection{Requirements}

The first task is to identify a suitable service to conduct a comprehensive analysis for the required adaptation, security, and relevant standards (shown as separate tasks in Figure 3). Typically, organisational services have different characteristics and requirements which make the analysis far from straightforward. Failure or inaccurate assessment in this step will result in the selection of inappropriate services that could cause complexity and integration issues. 
While many applications have already been developed specifically for the cloud environment, others must be adapted to be suitable for the cloud which involves making them cloud enabled (Andrikopoulos et al., 2013b). Adaptations may be required at a number of levels: data layer, data-base layer, accessibility, and business processes to enable existing applications to function in a multi-client and distributed environment. These adaptations will also ensure the acquirement of the main cloud features such as scalability and availability. However, many applications are not ready to be moved to the cloud because the environment is not mature enough for them e.g. safety-critical software (Binz et al., 2012).

Cloud security is a joint responsibility between cloud providers and users. The cloud users' responsibility starts by migrating the services that their security privileges can be addressed by the cloud vendors. Organisations must make sure that the appropriate authentication and identity management capabilities are integrated into the applications to protect and have access to encrypted data, particularly while data is transferred across cloud boundaries. Securing APIs and key management must also be ensured during the design phase of applications migration (CSA, 2013).

\subsubsection{Integration}

Enterprises are likely to have hybrid of cloud and in-houses systems, resulting in the assessment of integration of candidate services to ensure smooth interactions between cloud services and on-premises systems. Moreover, in some cases enterprises need to combine a range of cloud services from different cloud providers to achieve maximum efficiencies which require further assessment of integration (Baum et al., 2014). Assessment of integration requires relevant expertise in developing cloud-to-cloud, cloud-to-local integration mechanisms as well as cloud integration management. Further, the integration roles require the development of relevant skills within areas such as business processes, data management, data analysis, business architecture, and service oriented architecture.

\subsubsection{Data volume}

Organisations are required to measure their data volume as it has a direct impact on cost calculation. Usually, cloud computing is more cost effective for start-ups or newly started organisations. On the other hand, companies that have large legacy-data will find migration more difficult and more expensive. For these organisations, cloud services, would be more beneficial for the provision of new services but not to replace, or migrate and deploy services.

\subsubsection{Critical to business}

Although, cloud computing uses up-to-date security mechanisms, it has not yet reached the maturity level for migrating highly sensitive data. Therefore, organisations are recommended to analyse their services in terms of the type of data, and their impact on the organisation (the tasks shown in Figure 3 under Critical to business). Services that deal with highly sensitive data such as finance services usually have more stringent requirements, resulting in more difficulties during migration. Additionally, it is very likely that the transformation to the cloud will result in new jobs and change in many job descriptions. To manage the impact on staffing, organisations need to analyse the anticipated change to the staffing level, roles and expertise during the design phase, before migrating existing services to the cloud.

Adopting cloud computing requires a fundamental shift in organisational culture and business processes, both within and outside of IT. Organisations should develop a full understanding of the impact of migrating services to the cloud, bearing in mind the 
organisational culture and the resulting cultural shift. This will minimise the impact of the change (disruption) and allow the cloud culture to be integrated within the organisations. This includes institutional values, business processes, resources utilisation, knowledge sharing, the interaction between members monitoring and internal audit, user access provisioning, departments, and with other organisations. Organisations should start by migrating less critical services and then gradually move to critical ones.

\subsubsection{Return on Investment (ROI)}

For the majority of organisations, cost reduction is the main motivation for cloud migration. The investment for migration requires organisations to conduct evaluation on ROI that is the financial metric to estimate the financial outcome of the investment. Several factors are required to be considered when exploring ROI. They include the business benefits against extra costs such as the need to invest in higher bandwidth, integration and staff training. The time, efforts, and cost of moving the service back in-house or to another provider should also be considered during the analysis. The variety of cloud services and deployment models and their payments polices have increased the complexity of ROI. Additionally, organisations have different sets of requirements such as legal requirements and they are in different positions with regard to the maturity and the legacy of their existing systems. The other factor that needs to be considered during the evaluation of ROI is the cost of the organisational impacts.

\subsection{Risk assessment}

The second key step in the design phase is conducting a risk assessment for each candidate service. The extensive concerns about the risks of migrating to the cloud (discussed in 3.1) require thorough risk assessments that include a wide range of tasks. They should measure the magnitude of the potential losses and their probabilities by performing a number of tasks. These tasks are categorised into four main areas: risk to the organisation, legal implications, accessibility, and availability, as shown in Figure 3. This will allow cloud customers to determine the acceptable level with respect to each risk and to negotiate risk balancing or avoidance. The risks to the organisation may include: loss of control, dependability, managing relationships with different cloud providers, process disruption, liability, business continuity and disaster recovery, and performance monitoring. For example, if an organisation is unable to access cloud services at a critical time, it is largely dependent on the cloud provider's customer service to provide information, and a lack of such information can lead to poor communication with the service's users'. Legal implications may include: intellectual property rights, compliance with regulations, software licenses, and lack of standardisation. Increased distance, as a consequence of migration, can result in higher security risks and bring about changes with regard to accessibility and availability.

\subsection{Vendors evaluation and implementation}

After identifying a suitable service for migration, the next step is to evaluate the cloud vendors to select appropriate providers. In this model, the SMICloud (discussed in section 2.2) has been adopted for ranking and evaluation of cloud providers. It consists of six primary areas shown as tasks in Figure 3 that are needed by cloud customers for selecting a service provider. The Accountability attribute is used to measure specific characteristics of cloud providers to build the trust of a customer on a cloud provider by ensuring accountability of security exposures and compliance that includes a measurement of auditability, compliance, 
data ownership, provider ethicality, and sustainability. Agility is to measure how quickly new capabilities are integrated into IT as needed by the business. The assurance characteristic is monitored to ensure that the cloud service is performing as expected or as specified in the Service Level Agreement (SLA). Cost tends to be the most quantifiable attribute, but it is important to measure cost in such a way which is relevant to a particular business requirements. Cloud offers usually have different performance in terms of functionality, service response time and accuracy. Organisations need to understand how their applications will perform on the different cloud services and whether their deployments meet their expectations. Security and privacy are major concerns for the majority of organisations. They are multi-dimensional in nature and include many attributes such as protecting confidentiality and privacy, data integrity and availability.

The final step in this process is to make the choice of the most appropriate cloud provider and then implementation. The implementation step includes the tasks of training members of staff on the new system, communication of the new service's information to all affected parties, and then producing relevant documentation.

\section{Model evaluation}

The proposed model was evaluated by a group of cloud practitioners who provided their views on the model's attributes. 69 practitioners within three categories: those who had been involved in cloud migration projects, those planning to migrate to the cloud, and those conducting research within cloud computing participated in the evaluation. The evaluation aimed to determine the degree to which the model is an accurate representation for a systematic migration decision process. In particular, it aimed to validate the following:

- Was the order of the steps within the model correct to ensure a systematic migration decision?

- Were the identified steps fundamental during migration decision-making process?

- Were the tasks within the steps relevant to the migration challenges?

- Does the model overlook important tasks to be performed within the migration steps?

- Does the model reflect the challenges of migrating to the cloud?

- Finally, does the model aid potential cloud customers to decide whether' to migrate to the cloud?

The overall result shows that the participants agreed with the structure and the tasks identified within this model. The vast majority $(88 \%)$ stated that the model was an effective guide that can support cloud computing migration decisions. In addition, $84 \%$ of the respondents indicated that the model is an accurate reflection of the issues involved in migrating to the cloud. The analysis shows that a substantial number of responses highly rated the tasks related to assessing risks and security throughout the model. For example, in the second step acquiring information with regard to cloud security policies obtained an average rating of 4.71out of 5 compared to an overall average of 4.32 in the other five tasks that were identified in the same step. This validates the still continuing security concerns associated with migration to the cloud which this model tries to mitigate by including security and risk assessment tasks throughout the steps. To evaluate the model, the following sub-sections analyse the participants' responses to the main questions asked. 


\subsection{The order of the steps}

One of the main motivations for this research is to remedy the lack of a process that ensures a systematic migration decision. The participants were asked to rank the steps identified in the model that arranged in a random order. The question was: The following is a list of the steps within the model that organisations should go through for migrating to cloud computing. If you do not agree with the order, please indicate the order that you wish to see in the model. The analysis of the responses is presented in table 5.

Table 5 The order of the steps

\begin{tabular}{|c|c|c|c|c|c|c|}
\hline Steps ${ }^{2}$ Order & 1 & 2 & 3 & 4 & 5 & 6 \\
\hline $\begin{array}{l}\text { Define the } \\
\text { business } \\
\text { strategy }\end{array}$ & $\begin{array}{c}87.50 \% \\
56\end{array}$ & $\begin{array}{c}4.69 \% \\
3\end{array}$ & $\begin{array}{c}1.56 \% \\
1\end{array}$ & $\begin{array}{l}3.13 \% \\
2\end{array}$ & $\begin{array}{c}0.0 \% \\
0\end{array}$ & $\begin{array}{c}3.13 \% \\
2\end{array}$ \\
\hline $\begin{array}{l}\text { Identify the } \\
\text { cloud } \\
\text { principles and } \\
\text { capabilities }\end{array}$ & $\begin{array}{c}4.69 \% \\
3\end{array}$ & $\begin{array}{c}71.88 \% \\
46\end{array}$ & $\begin{array}{c}17.19 \% \\
11\end{array}$ & $\begin{array}{c}4.69 \% \\
3\end{array}$ & $\begin{array}{c}1.56 \% \\
1\end{array}$ & $\begin{array}{c}\mathbf{0 . 0} \% \\
0\end{array}$ \\
\hline $\begin{array}{l}\text { Identify the } \\
\text { suitable } \\
\text { services for } \\
\text { the cloud } \\
\text { environment }\end{array}$ & $\begin{array}{c}7.81 \% \\
5\end{array}$ & $\begin{array}{c}9.38 \% \\
6\end{array}$ & $\begin{array}{l}64.06 \% \\
41\end{array}$ & $\begin{array}{c}6.25 \% \\
4\end{array}$ & $\begin{array}{c}12.50 \% \\
8\end{array}$ & $\begin{array}{c}\mathbf{0 . 0} \% \\
0\end{array}$ \\
\hline $\begin{array}{l}\text { Conduct risk } \\
\text { assessment }\end{array}$ & $\begin{array}{c}0.0 \% \\
0\end{array}$ & $\begin{array}{c}10.94 \% \\
7\end{array}$ & $\begin{array}{c}9.38 \% \\
6\end{array}$ & $\begin{array}{c}40.63 \% \\
26 \\
\end{array}$ & $\begin{array}{c}35.94 \% \\
23 \\
\end{array}$ & $\begin{array}{l}3.13 \% \\
2\end{array}$ \\
\hline $\begin{array}{l}\text { Evaluate } \\
\text { venders }\end{array}$ & $\begin{array}{c}\mathbf{0 . 0} \% \\
0\end{array}$ & $\begin{array}{c}1.56 \% \\
1 \\
\end{array}$ & $\begin{array}{c}4.69 \% \\
3\end{array}$ & $\begin{array}{c}45.31 \% \\
29 \\
\end{array}$ & $\begin{array}{c}46.88 \% \\
30 \\
\end{array}$ & $\begin{array}{c}1.56 \% \\
1\end{array}$ \\
\hline Implementation & $\begin{array}{c}0.0 \% \\
0\end{array}$ & $\begin{array}{c}1.56 \% \\
1\end{array}$ & $\begin{array}{l}3.13 \% \\
2\end{array}$ & $\begin{array}{c}\mathbf{0 . 0 \%} \\
0\end{array}$ & $\begin{array}{c}3.13 \% \\
2\end{array}$ & $\begin{array}{c}92.19 \% \\
59\end{array}$ \\
\hline
\end{tabular}

It can be seen that there is a high level of agreement with the model structure. In particular, the participants agreed strongly with the order of the first three steps and the last one. However, about $35.9 \%$ would have placed the risk assessment in the fifth step (after the vendor evaluation step) compared to $40.6 \%$ who would have placed it in the fourth step. The participants who preferred this sequence (that is, provider evaluation prior to risk assessment) did so largely because they would prefer to include the vendor evaluation within the risk assessment. However, the risk assessment step in this model is within the design phase to ensure service suitability for migration to the cloud environment. In addition, the vendor evaluation step includes a number of tasks that assess the potential risks.

In a separate question, the participants were asked to rate the importance of the steps. The risk assessment step was rated most highly with an average rating of 4.68 out of 5. Evaluating organisations' requirements and Defining the business strategy came second with an average rating of 4.55. The average rates of the other steps were as follows: Evaluating vendors, 4.33; Identifying suitable services for the cloud environment, 4.26; and identifying the cloud guiding principle and capabilities, 4.18 respectively.

\subsection{The tasks within the cloud environment step}

Table 6 shows the analysis of evaluating the importance of the tasks within the cloud environment steps. It shows strong agreement with the need to acquire a wide range of information about the new environment. Because the largest standard deviation (0.83) is less than twice the smallest $(2 * 0.59=1.2)$, the data look reasonably normal and the assumption of equal standard deviation can be used. Analysis Of Variance (ANOVA) test was performed on the data that displays an $\mathrm{F}$ value of (4.92) which is greater than the critical value (2.23) as shown in Table 6 . Therefore, the null hypothesis can be rejected (that there is no difference between the ratings of the tasks). This result supports conclusions to be drawn based on a comparison of means. 
With an average rating of 4.32 out of 5, the participants acknowledged the areas identified within this step. This average is the highest in comparison to the averages of the tasks within the other steps in this model. Being aware of the security policies within the cloud environment was rated highest with (95.59) of respondents rating it high or very high. Compliance with regulations also scored highly with (89.56) of participants rating it high or very high, while identifying the level of support scored the least with an average rate of 4.17.

Table 6 The tasks within the cloud environment step

\begin{tabular}{|c|c|c|c|c|c|c|c|c|}
\hline Tasks & 1 & 2 & 3 & 4 & 5 & Average & Variance & Std Dev \\
\hline Cloud services management & 0 & $1.47 \%$ & $19.12 \%$ & $35.29 \%$ & $44.12 \%$ & 4.220588 & 0.652107 & 0.807531 \\
\hline Pricing policies & 0 & $2.94 \%$ & $11.76 \%$ & $38.24 \%$ & $47.06 \%$ & 4.294118 & 0.628622 & 0.792857 \\
\hline Security polices & 0 & $1.47 \%$ & $2.94 \%$ & $19.12 \%$ & $76.47 \%$ & 4.705882 & 0.359965 & 0.599971 \\
\hline Performance monitoring & 0 & $1.47 \%$ & $19.12 \%$ & $42.65 \%$ & $36.76 \%$ & 4.147059 & 0.604917 & 0.777764 \\
\hline Compliance to regulators & 0 & $2.99 \%$ & $7.46 \%$ & $38.81 \%$ & $50.75 \%$ & 4.373134 & 0.570782 & 0.755501 \\
\hline Support & 0 & $4.48 \%$ & $13.43 \%$ & $41.79 \%$ & $40.30 \%$ & 4.179104 & 0.694708 & 0.833492 \\
\hline \multicolumn{9}{|l|}{ ANOVA } \\
\hline Source of Variation & \multicolumn{2}{|c|}{ SS } & \multicolumn{2}{|r|}{$d f$} & MS & $F$ & P-value & F crit \\
\hline Between Groups & \multicolumn{2}{|c|}{14.39611382} & \multicolumn{2}{|r|}{5} & 2.879223 & \multirow[t]{2}{*}{4.922205} & \multirow[t]{2}{*}{0.00022} & 2.236551 \\
\hline Within Groups & \multicolumn{2}{|c|}{233.9782704} & \multicolumn{2}{|c|}{400} & 0.584946 & & & \\
\hline Total & \multicolumn{2}{|c|}{248.3743842} & \multicolumn{2}{|c|}{405} & & & & \\
\hline
\end{tabular}

\subsection{The tasks within the service suitability step}

In a question (see Table 7) the participants were asked to rate the importance of the tasks identified in the Service suitability step. The largest standard deviation (1.03) is less than twice the smallest $(2 * 0.67=1.35)$ which indicates that the data are reasonably normal. Based on ANOVA, an F value of (9.88) is greater than the critical value (2.02) as shown in Table 7. This supports conclusions to be drawn based on a comparison of the means.

With an average rating of 4.02 out of 5, the results show the respondents' level of agreement with the tasks identified within this step. Similar to the Cloud environment step, security requirements task was seen by the participants as the most important factor when selecting a service to be migrated. It obtained an average rating of 4.49 respectively while the impact on an organisation's culture and staff scored the lowest rating of 3.49. As expected, the Return on Investment (ROI) calculation task was highly rated with an average rating of 4.25. Identifying the integration requirements task was also highly rated in this step, with an average rating of 4.22 .

Table 7 The tasks within the service suitability step

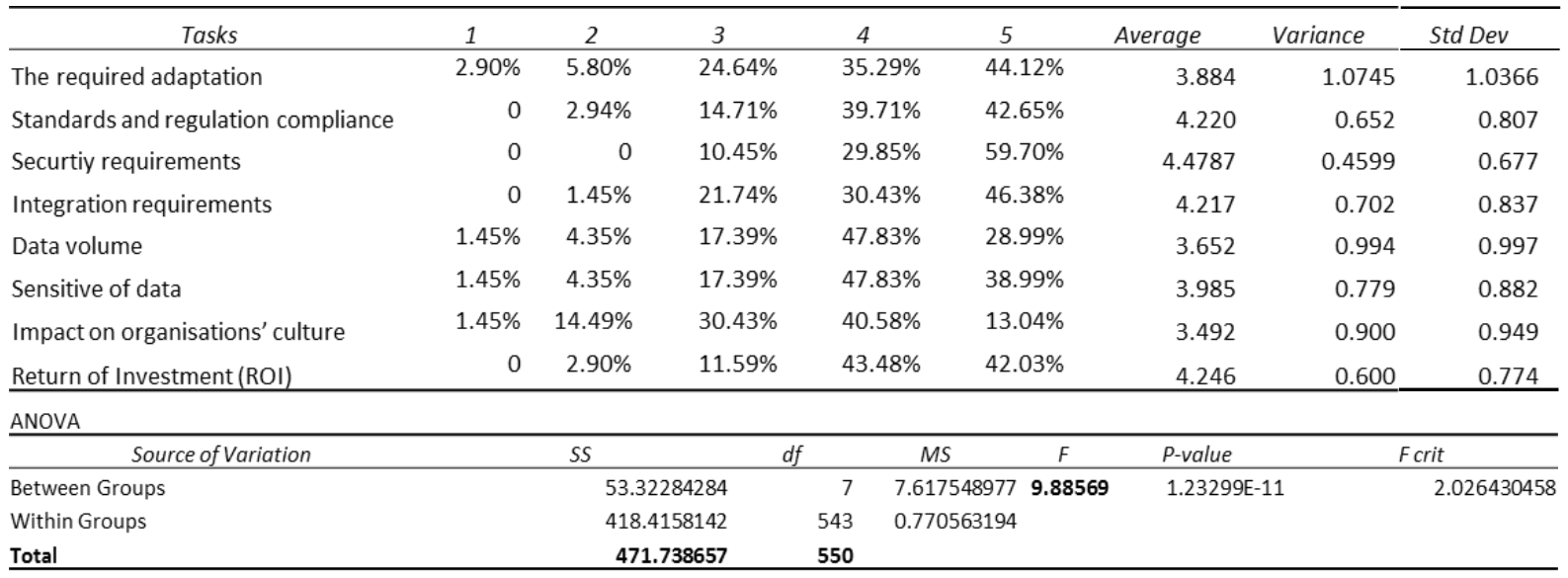




\subsection{The tasks within the risk assessment step}

In a question (see table 8), the participants were asked to rate the importance of tasks to be performed within the Risk assessment step for each candidate service. The participants were given a list of 17 tasks which were categorised into four main areas in the model.

Table 8 The tasks within the risk assessment step

\begin{tabular}{|c|c|c|c|c|c|c|c|c|}
\hline Tasks & 1 & 2 & 3 & 4 & 5 & Average & Variance & Std Dev \\
\hline Loss of control & 0 & $1.45 \%$ & $17.39 \%$ & $34.78 \%$ & $46.38 \%$ & 4.261 & 0.637 & 0.798 \\
\hline Dependability assessment & 0 & $8.82 \%$ & $17.56 \%$ & $38.24 \%$ & $35.29 \%$ & 3.986 & 0.897 & 0.947 \\
\hline Managing relationship with diffe cloud providers & $4.41 \%$ & $8.82 \%$ & $17.65 \%$ & $38.24 \%$ & $35.29 \%$ & 3.559 & 1.385 & 1.177 \\
\hline Process disruption evaluation & 0 & $7.25 \%$ & $28.99 \%$ & $39.24 \%$ & $27.54 \%$ & 3.841 & 0.842 & 0.918 \\
\hline Service level and performance monitoring & 0 & $2.94 \%$ & $16.18 \%$ & $54.41 \%$ & $26.47 \%$ & 4.029 & 0.558 & 0.747 \\
\hline Privacy and data confidentiality & 0 & 0 & $15.94 \%$ & $28.99 \%$ & $55.07 \%$ & 4.362 & 0.646 & 0.804 \\
\hline Auditing & 0 & $725 \%$ & $27.54 \%$ & $33.33 \%$ & $31.88 \%$ & 3.913 & 0.875 & 0.935 \\
\hline Liability & $1.45 \%$ & $4.35 \%$ & $34.78 \%$ & $33.33 \%$ & $29.09 \%$ & 3.768 & 0.887 & 0.942 \\
\hline Response times & 0 & $10.14 \%$ & $28.99 \%$ & $40.58 \%$ & $20.29 \%$ & 3.725 & 0.820 & 0.906 \\
\hline Business continuity and disaster recovery & 0 & $2.90 \%$ & $17.39 \%$ & $43.48 \%$ & $36.23 \%$ & 4.145 & 0.655 & 0.809 \\
\hline Flexibility of service & $2.90 \%$ & $8.70 \%$ & $27.54 \%$ & $34.78 \%$ & $26.09 \%$ & 3.725 & 1.026 & 1.013 \\
\hline Compliance with regulations & 0 & $2.90 \%$ & $15.94 \%$ & $39.13 \%$ & $42.03 \%$ & 4.217 & 0.673 & 0.820 \\
\hline Ease of ending the arrangement (termination) & 0 & $11.59 \%$ & $20.29 \%$ & $43.48 \%$ & $24.64 \%$ & 3.797 & 0.899 & 0.948 \\
\hline Data ownership and IP rights & 0 & $1.45 \%$ & $10.14 \%$ & $43.48 \%$ & $44.93 \%$ & 4.279 & 0.533 & 0.730 \\
\hline Legal implication & 0 & $1.45 \%$ & $15.94 \%$ & $42.03 \%$ & $40.58 \%$ & 4.206 & 0.584 & 0.764 \\
\hline Accessibility & 0 & 0 & $15.94 \%$ & $46.38 \%$ & $37.68 \%$ & 4.206 & 0.494 & 0.703 \\
\hline Availability & 0 & 0 & $14.49 \%$ & $42.03 \%$ & $43.48 \%$ & 4.299 & 0.485 & 0.697 \\
\hline \multicolumn{9}{|l|}{ ANOVA } \\
\hline Source of Variation & $d f$ & & MS & \multicolumn{2}{|r|}{$F$} & P-value & \multicolumn{2}{|c|}{ Fcrit } \\
\hline Between Groups & & 16 & \multirow{2}{*}{\multicolumn{2}{|c|}{$\begin{array}{l}4.232947982 \\
0.759039078\end{array}$}} & 5.576719436 & \multirow[t]{2}{*}{$1.11968 \mathrm{E}-11$} & \multirow{2}{*}{\multicolumn{2}{|c|}{1.652308}} \\
\hline Within Groups & & 1150 & & & & & & \\
\hline 940.622108 & & 1166 & & & & & & \\
\hline
\end{tabular}

The largest standard deviation (1.03) is less than twice the smallest $(2 * 0.67=1.35)$ that shows a normality of the data. An F value of (9.88) (ANOVA based analysis) is greater than the critical value (2.02) as shown in Table 7. This supports conclusions to be drawn based on comparing the means.

Privacy and data confidentiality, followed by data ownership and intellectual property rights, were rated most highly among these tasks with average rates of 4.39 and 4.32 respectively. The availability and accessibility assessment tasks were also highly rated at 4.22 and 4.29 respectively. Similarly, the Legal risk assessment tasks were highly rated. Assessing the potential legal implication obtained an average rating of 4.22 and compliance with regulations obtained an average rating of 4.20 .

In separate questions, the tasks identified within the last two steps of the model scored average ratings of 4.34 and 4.09 respectively. In the vendor evaluation step, Security and privacy (4.77), followed by Performance (4.52) were chosen by the participants as the main factors when selecting a service provider. The tasks in the Implementation step were rated as follows: need for staff training, 4.12; communication, 4.16; and documentation, 3.99.

\section{Conclusion}

Migration to cloud computing is a strategic organisational decision that is complicated, dynamic, and highly unstructured, due to the immaturity and the still evolving nature of the cloud environment. This has intensified the requirement for a thorough process that identifies the main issues to be considered and the tasks to be performed, to ensure that informed decisions are made for migration to the cloud.

In this paper, the existing cloud DSSs were evaluated. They mostly provide information to support the evaluation and selection of vendors with cost being the main factor, while many fundamental issues had been left unsupported. They ignored providing information about the 
cloud environment and also offered limited support to assess the suitability of the cloud environment to replace the in-house services. Despite the increase in research into support for migration decisions, there is still a lack of tools that automate the migration tasks.

In this research, A two-stage survey was employed that identified the challenges faced by decision makers and the activities needed to be performed. A generic decision-making model was utilised to develop a model to support the decision making process for migration to the cloud. The model includes relevant activities, phases, steps and tasks.

The unique contribution of this paper is the capability of the proposed model to systematically cover all the stages required for making decisions when considering migration to the cloud. The model includes three phases for decision making: intelligence design and choices. These phases are expanded into six key steps, each with a number of tasks to be performed. The steps and tasks can be performed in conjunction with the information that resides in the knowledge-base. The model incorporates intelligence tasks into the tasks required for services suitability and vendors evaluation, especially the tasks in cloud environment step which can be very helpful for customers who are not familiar with it.

The model was evaluated, using the views of a group of the cloud practitioners. The model was seen by practitioners as an effective guide for decision makers to make informed decisions for migrating to the cloud. The analysis of the views demonstrated a high level of acceptance by the practitioners with regard to the structure, tasks, and issues addressed by the model. Although the model was evaluated, the unavailability of a DSS for real testing and implementation of the model is a limitation of this study.

One of the key features of the model is the use of cloud DSSs. The model can enable decision makers to effectively exploit a wide range of the existing cloud migration DSSs. The model will reduce the amount of effort required for information gathering, suitability analysis, and vendor's evaluations. It enhances the understanding of decisions made, and improved the efficiency of the migration process cycle. It offers a preliminary structure for developing a cloud KBDSS. KBDSSs can address many of the challenges of migration process. Future work will be necessary to develop a framework for integrating the required cloud DSSs and tools with knowledge management processes including, discovery and detection of trends and patterns, knowledge acquisition, application, and sharing.

\section{References}

Ada, Ş., Karaman, E., and Ghaffarzadeh, M. (2015) 'Decision Making Based on Management Information System and Decision Support System', Journal for Studies in Management and Planning, Vol. 1, No. 3, pp. 206-216.

Aggarwal, V., Mathur, M., and Saraswat, N. (2013) 'Comprehensive Cloud Incremental DataApplication Migration-A Proposed Model for Cloud Migration' International Journal of Computer Applications in Engineering Sciences, Vol. 3, No. 1, pp. 22.

Alkhalil, A., Sahandi, R., and John, D. (2013) 'Migration to Cloud Computing-The Impact on IT Management and Security', 1st International Workshop on Cloud Computing and Information Security, Atlantis Press.

Alkhalil, A., Sahandi, R., and John, D. (2014) 'Migration to Cloud Computing: A Decision Process Model', $25^{\text {th }}$ international conference on information and intelligent systems, faculty of organisation and informatics, Varazdin, Croatia.

Al-Roomi, M., Al-Ebrahim, S., Buqrais, S., and Ahmad, I. (2013) 'Cloud Computing Pricing Models: A Survey’ International Journal of Grid \& Distributed Computing, Vol. 6, No. 5, pp. 93-106. 
Andrikopoulos, V., Song, Z., and Leymann, F. (2013a) 'Supporting the Migration of Applications to the Cloud through a Decision Support System', Sixth IEEE International Conference on Cloud Computing, pp. 565-572.

Andrikopoulos, V., Binz, T., Leymann, F., and Strauch, S. (2013b) 'How to adapt applications for the Cloud environment', Computing, Vol. 95, No. 6, pp. 493-535.

Baum, D., Raheja, R., Tierney, B., and Pawar, V. (2014) 'Cloud Integration - A Comprehensive Solution', [online] http://www.oracle.com/us/solutions/cloud/cloudintegration-wp-1873149.pdf, (Accessed 01 May 2014).

Beserra, P. V., Camara, A., Ximenes, R., Albuquerque, A. B., and Mendonca, N. C. (2012) 'Cloudstep: A step-by-step decision process to support legacy application migration to the cloud', 6th International Workshop on the (MESOCA), IEEE, pp. 7-16.

Binz, T., Breiter, G., Leyman, F., and Spatzier, T. (2012) 'Portable Cloud Services Using TOSCA.', IEEE Internet Computing, Vol.16,No. 3.

Cloud Standards Coordination (CSC). (2013) [online] http://www.etsi.org/images/files/Events/2013/2013_CSC_Delivery_WS/CSCFinal_report-013-CSC_Final_report_v1_0_PDF_format-.PDF, (Accessed 24 June 2014).

CSA. (2013) 'Practices for Secure Development of Cloud' Cloud security Alliance [online] http://www.safecode.org/publications/SAFECode_CSA_Cloud_Final1213.pdf, (Accessed 10 July 2013).

Dhinesh Babu, L. D., Gunasekaran, A., and Krishna, P. V. (2014) 'A decision-based preemptive fair scheduling strategy to process cloud computing work-flows for sustainable enterprise management', International Journal of Business Information Systems, Vol. 16, No.4, pp. 409-430.

Diniz VB., Borges MRS, Gomes JO., Canos JH. (2008) 'Decision making support in emergency response', Encyclopedia of decision making and decision support technologies, No. 1, pp. 184-191.

García-Galán, J., Trinidad, P., Rana, O. F., and Ruiz-Cortés, A. (2015) ‘Automated configuration support for infrastructure migration to the cloud', Future Generation Computer Systems.

Garg, S. K., Versteeg, S., and Buyya, R. (2013) 'A framework for ranking of cloud computing services’, Future Generation Computer Systems, Vol. 626, No. 4, pp. 1012-1023.

Gleeson N. and Walden, I. (2014) 'It's a jungle out there'?: Cloud computing, standards and the law', European Journal of Law and Technology, Vol. 5, No. 2.

Gonidis, F., Paraskakis, I., and Simons, A. J. (2014) 'Leveraging Platform Basic Services in Cloud Application Platforms for the Development of Cloud Applications', IEEE 6th International Conference on Cloud Computing Technology and Science pp. 751-754.

Iveroth, E., Westelius, A., Petri, C. J., Olve, N. G., Cöster, M., and Nilsson, F. (2013) 'How to differentiate by price: Proposal for a five-dimensional model', European Management Journal, Vol. 32, No. 2, pp. 109-123.

Jamshidi, P., Aakash A., and Claus, P. (2013) 'Cloud migration research: a systematic review', IEEE Transactions on Cloud Computing,Vol. 1, No. 2, pp. 142-157.

Khajeh-Hosseini, A., Greenwood, D., Smith, J. W., and Sommerville, I. (2012) 'The cloud adoption toolkit: supporting cloud adoption decisions in the enterprise' Software: Practice and Experience, Vol. 42, No. 4, pp. 447-465.

Meng, S., Iyengar, A. K., Rouvellou, I. M., Liu, L., Lee, K., Palanisamy, B., and Tang, Y. (2012) 'Reliable state monitoring in cloud datacenters', 5th International Conference on Cloud Computing, IEEE, pp. 951-958. 
Menzel, M., Ranjan, R., Wang, L., Khan, S., and Chen, J. (2014) 'Cloudgenius: A hybrid decision supportmethod for automating the migration of webapplication clusters to public clouds', IEEE transactions on, Vol. 64, No. 5.

Misra, S. C., and Mondal, A. (2011) 'Identification of a company's suitability for the adoption of cloud computing and modelling its corresponding Return on Investment', Mathematical and Computer Modelling, Vol. 53, No. 3, pp. 504-521.

Omerovic, A., Muntes-Mulero, V., Matthews, P., and Gunka, A. (2013) 'Towards a Method for Decision Support in Multi-cloud Environments', The Fourth International Conference on Cloud Computing, GRIDs, and Virtualization, pp. 244-250.

Rai, R., Mehfuz, S., and Sahoo, G. (2014) 'Efficient Migration of Application to Clouds: Analysis and Comparison', Journal on Computing (JoC), Vol. 3, No. 3.

Sahandi, R., Alkhalil, A., and Opara-Martins, J. (2013) 'Cloud computing from SMEs perspective: a survey based investigation', Journal of Information Technology Management, Vol. 24, No. 1, pp. 2.

Santiago, G (2015) 'Performance and Cost Evaluation for the Migration of a Scientific Workflow Infrastructure to the Cloud', $5^{\text {th }}$ International Conference of Cloud Computing and Service Science, CLOSER 2015, Lisbon, Portugal, 20-22 May 2015.

Sharma, A. and Abrol, P. (2013) 'Cloud Computing Environment: Problems in Implementation' International Journal of Computers and Distributed Systems, Vol. 3, No.1

Simon, H. A. (1977) 'The new science of management decision', $2^{\text {nd }}$ Edition, PrenticeHall, Englewood Cliffs (NJ). 